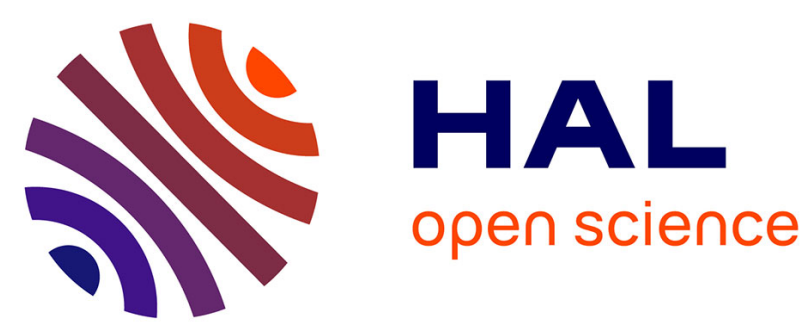

\title{
Directional Fano resonances in light scattering by a high refractive index dielectric sphere
}

Michael I. Tribelsky, Jean-Michel Geffrin, Amelie Litman, Christelle Eyraud, Fernando Moreno

\section{- To cite this version:}

Michael I. Tribelsky, Jean-Michel Geffrin, Amelie Litman, Christelle Eyraud, Fernando Moreno. Directional Fano resonances in light scattering by a high refractive index dielectric sphere. Physical Review B: Condensed Matter and Materials Physics (1998-2015), 2016, 94 (12), pp.121110. 10.1103/PhysRevB.94.121110 . hal-01365811

\section{HAL Id: hal-01365811 \\ https://hal.science/hal-01365811}

Submitted on 13 Sep 2016

HAL is a multi-disciplinary open access archive for the deposit and dissemination of scientific research documents, whether they are published or not. The documents may come from teaching and research institutions in France or abroad, or from public or private research centers.
L'archive ouverte pluridisciplinaire HAL, est destinée au dépôt et à la diffusion de documents scientifiques de niveau recherche, publiés ou non, émanant des établissements d'enseignement et de recherche français ou étrangers, des laboratoires publics ou privés. 


\title{
Directional Fano resonances in light scattering by a high refractive index dielectric sphere
}

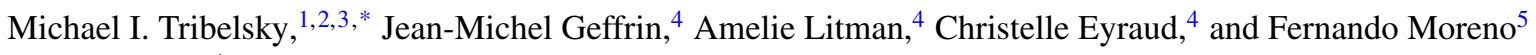 \\ ${ }^{1}$ Faculty of Physics, M. V. Lomonosov Moscow State University, Moscow 119991, Russia \\ ${ }^{2}$ Moscow Technological University MIREA, Moscow 119454, Russia \\ ${ }^{3}$ National Research Nuclear University MEPhI (Moscow Engineering Physics Institute), Moscow 115409, Russia \\ ${ }^{4}$ Aix Marseille Univ, CNRS, Centrale Marseille, Institut Fresnel, Marseille, France \\ ${ }^{5}$ Group of Optics, Department of Applied Physics, University of Cantabria, Cantabria, Spain
}

(Received 21 May 2016; revised manuscript received 15 August 2016; published 12 September 2016)

\begin{abstract}
We report the experimental evidence of directional Fano resonances at the scattering of a linearly polarized electromagnetic plane wave by a homogeneous dielectric sphere with a high refractive index and low losses. We observe a typical asymmetric Fano profile for the intensity scattered in practically any given direction, while the overall extinction cross section remains Lorentzian. The phenomenon originates in the interference of the selectively excited electric dipolar and quadrupolar modes. The selectivity of the excitation is achieved by the proper choice of the frequency of the incident wave. Owing to the scaling invariance of the Maxwell equations, in these experiments we mimic the scattering of the visible and near IR radiation by a nanoparticle made of common semiconductor materials ( $\mathrm{Si}, \mathrm{Ge}, \mathrm{GaAs}, \mathrm{GaP}$ ) by the equivalent scattering of a spherical particle of $18 \mathrm{~mm}$ in diameter in the microwave range. The theory developed to explain the experiments extends the conventional Fano approach to the case when both interfering partitions are resonant. A perfect agreement between the experiment and the theory is demonstrated.
\end{abstract}

DOI: 10.1103/PhysRevB.94.121110

Introduction. Light scattering by particles is a rather old topic, but it still remains one of the most important issues of electrodynamics. Nowadays, interest in this problem is more pronounced than ever. On one hand, it is explained by numerous applications of the phenomenon in nanotechnologies, telecommunications, medicine, biology and bioengineering, chemistry, etc. [1]. On the other hand, it is stimulated by the discovery of some very unusual properties of light scattering. Among them, Fano resonances [2,3] should be mentioned. The resonances are explained by the presentation of a scattered wave as a sum of two partitions - a weakly $\omega$-dependent background and a sharply $\omega$-dependent resonant one, where $\omega$ stands for the frequency of the incident wave. Interference of the partitions results in a typical asymmetric Fano line with a sharp dependence of the overall scattering cross section on $\omega$.

However, for some applications (especially in telecommunications, information processing, optical tweezers, etc.) it is highly desirable to have a Fano profile for the intensity scattered in a given direction. Then, one obtains a tuned and controlled redistribution of scattered radiation in this direction and/or sharp spatial gradients of the scattered field. It may be achieved by means of directional Fano resonances, theoretically predicted in Ref. [4] for the Mie scattering by a sphere. In these resonances the role of a background partition is played by an off-resonant part of a multipole, while a sharp resonance line of another multipole represents a resonant partition. Since in the Mie expansion different multipoles have different angular dependences of the scattered radiation, at a given $\omega$ the Fano conditions may be satisfied along certain directions only. Regarding the overall scattering intensity, its profile remains symmetric Lorentzian.

Despite the apparent importance of the phenomenon, its experimental evidence has not been obtained for quite a

*tribelsky@mirea.ru while. The point is that the initial theory was developed in Ref. [4] for metal particles. Most metals are very lossy at the optical frequencies, and the discussed effect is suppressed by dissipation. As for generalizations of the theory to a dielectric scatterer, they have begun to be discussed only recently [5].

Ultimately, an experimental observation of the directional Fano resonances was reported [6]. However, the Fano profiles measured in Ref. [6] correspond to light scattering by a "metamolecule", consisting of nanospheres situated close to each other. They are related to the interference of the hybrid modes, which originate in the coupling of the spheres. Regarding the directionality, only the forward and backward scattering is studied. Thus, these results actually correspond to the manifestation of the Kerker effect [7] by a composite scatter. In contrast, according to Ref. [4], directional Fano resonances are a generic, intrinsic feature of light scattering by a single homogeneous particle with a simple shape (sphere, cylinder) and low losses. They are related to the excitation of conventional Mie modes and, what is most important, the Fano profile may be observed in any given direction. All these predictions have not yet been confirmed experimentally. The present Rapid Communication is an attempt to fill that gap.

Specifically, we inspect the scattering of a linearly polarized electromagnetic plane wave by a dielectric sphere with a high refractive index (HRI) and low losses. To avoid difficulties in precise measurements at the nanoscale, we employ a microwave analogy [8-10], mimicking the light scattering by a nanosphere made of a common semiconductor with a homogeneous, $18 \mathrm{~mm}$ diameter sphere composed of a special ceramic [11]. The selected range of problem parameters makes it possible to excite selectively the electric dipolar and quadrupolar modes, whose interference produces directional Fano resonances. We measure the line shape of the scattered intensity in a given direction and obtain pronounced typical Fano profiles for practically any scattering angle. We also measure the line shape of the net extinction cross section 
(which in our case is very close to the scattering one owing to the weak dissipation) and show that in the frequency range where the directional intensities exhibit Fano profiles, the former is Lorentzian. Finally, analyzing the exact Mie solution, we explain all the obtained experimental results analytically, extending the results of Ref. [4] to the problem in question. In particular, we show that in this case the line shape may be presented as a sum of two profiles, conventional Fano and Lorentzian. The parameters of these profiles have different dependences on the scattering angle, which provides an additional opportunity to tailor and engineer the desired line shape by simply varying the scattering angle.

The problem formulation. According to the exact Mie solution, describing the scattering, the scattered field is presented as an infinite series of partial waves (multipoles). Each multipole itself is a sum of two modes, electric and magnetic $[12,13]$. Thus, the scattering efficiency $Q_{\text {sca }}$ (the dimensionless ratio of the scattering cross section $C_{\text {sca }}$ to the geometric one $\pi R^{2}$, where $R$ stands for the radius of the sphere) equals

$$
Q_{\mathrm{sca}}=\sum_{\ell=1}^{\infty}\left(Q_{\mathrm{sca}, \ell}^{(a)}+Q_{\mathrm{sca}, \ell}^{(b)}\right) .
$$

Here, superscripts $(a)$ and $(b)$ designate the electric and magnetic contributions, respectively. Each partial efficiency, regarded as a function of $\omega$, has an infinite sequence of Mie resonances.

The dramatic difference between these resonances for a particle with a moderate value of refractive index and those with HRI is that, in the former, the resonance lines for different multipoles overlap substantially. In the latter, the overlap is much weaker. In our experiments, at the working frequency range $(4-8 \mathrm{GHz})$, the complex permittivity of the sphere, measured by the procedure described in Ref. [14], may be regarded as a constant, $\varepsilon=\varepsilon^{\prime}+i \varepsilon^{\prime \prime}=17.2+0.2 i$. This is a typical value for common semiconductors ( $\mathrm{Si}, \mathrm{Ge}, \mathrm{GaAs}, \mathrm{GaP}$ ) in the visible and near IR ranges of the spectrum [15]. The corresponding scattering efficiencies, calculated according to the exact Mie solution, are presented in Fig. 1, where $x=k R$ is the size parameter, $k=2 \pi / \lambda$, and $\lambda$ stands for the wavelength of the incident wave in a vacuum.

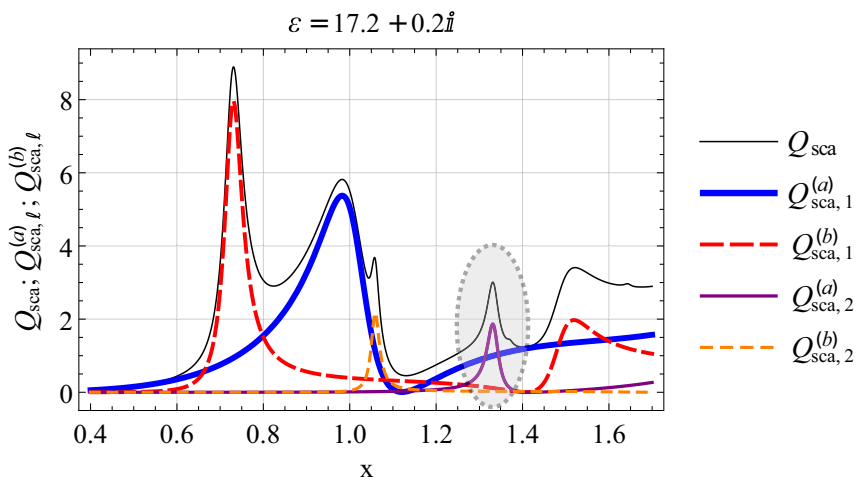

FIG. 1. The overall scattering efficiency $Q_{\text {sca }}$ and the first two (dipolar and quadrupolar) partial efficiencies at $\varepsilon=17.2+0.2 i$ according to the size parameter $x$.
The weak overlap of the resonance lines makes it possible to selectively excite the desired modes. In our recent study [16], we have taken this opportunity to create tunable scattering diagrams. Here, we will focus on the area, marked in Fig. 1 with an oval, where just the two modes, electric dipolar and quadrupolar, make a key contribution to the overall scattering. It is important that while at the edges of the marked area the quadrupolar efficiency is smaller than the dipolar one, in the middle of it, the case is opposite. In addition, the dipolar mode in this area is slowly varying (the background partition), while the quadrupolar mode varies sharply (the resonance partition). Such a behavior provides all the prerequisites for the manifestation of directional Fano resonances [4].

Experimental setup and results. For the experimental study of the phenomenon, we employ the bistatic facility in the anechoic chamber of the Centre Commun de Ressources en Microondes (CCRM). Our measurement protocol is rather common in radar cross-section experiments, with the measurement of a reference target, a background subtraction, and a software time gating. The measurements are performed with the emitting and receiving antennas both located in the azimuthal plane. These two horn antennas are linearly polarized and two polarization cases are measured, with either the emitter and the receiver polarization vectors parallel $(\mathrm{P})$ to the scattering (azimuthal) plane or perpendicular $(\mathrm{S})$ to it. A more detailed description of the facility and of its performances can be found in Refs. [9,14,17,18]. Such performances are rendered possible owing to the two main items that should be pointed out in our measurement protocol.

First, we employ a large angular range of our measurements (see Fig. 2) to enhance the postprocessing data treatment. Since the scattered field is obtained from the difference between the two fields (the net field measured by the receiver, when the scattering sphere is situated in the chamber, minus the incident field measured, when the sphere is removed), the result is very sensitive to any kind of disturbance (e.g., drifts, which necessarily appear during the measurements, etc.). To compensate them, we take advantage of the angular spectral properties of the scattered field [19] in the postprocessing step [20].

Second, as our measurements are referenced using a perfectly known target, they are calibrated so that the presented values are all quantitative, obtained in dimensional units, in contrast to conventional experiments in this field, whose results are presented in arbitrary units. It makes it possible to compare our measurements with the calculations based on the exact Mie solution quantitatively, while in the conventional approach only the line shapes may be compared.

Once the calibrations and the postprocessing treatments of the complex measured scattered field have been done, the extinction cross section may be obtained by means of the optical theorem [12]. To this end, we extract from the amplitude and phase of the electric field its value in the exact forward direction [21].

The results of these measurements and their comparison with the Mie solution are shown in Fig. 3. The extinction cross section may be independently extracted from the forward scattering with any of the two polarizations. We have overplotted the two independently extracted values, demonstrating the very good reproducibility of our measurements. A pronounced 

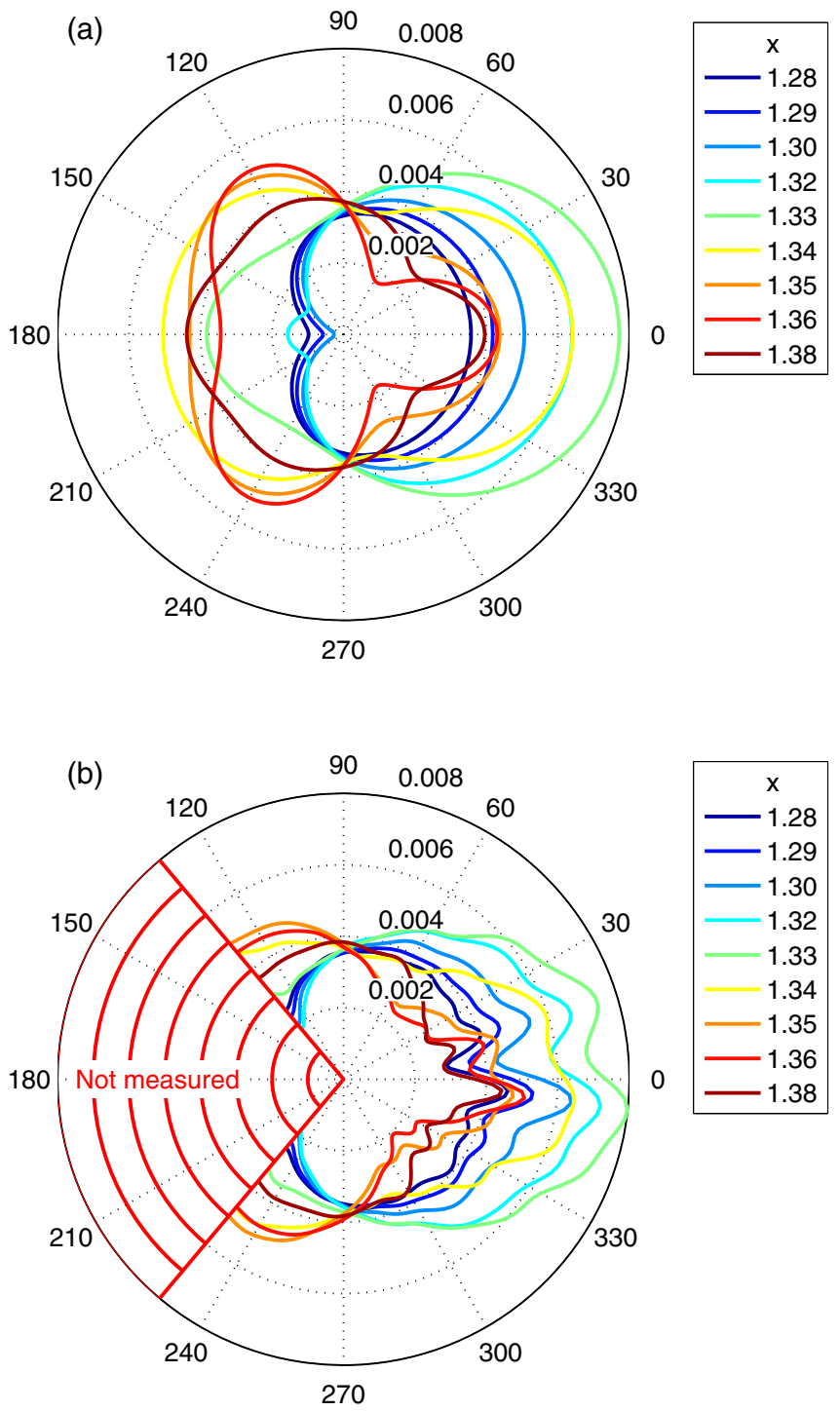

FIG. 2. Angular variations of the magnitude of the scattered electric field in the $\mathrm{S}$ polarization case. (a) Simulation according to the exact Mie solution. (b) Experimentally measured.

set of Fano profiles with various values of the asymmetry parameter as well as excellent quantitative agreement between the theory and experiment are seen straightforwardly. Note that the extinction cross section has an almost perfect Lorentzian shape. A small secondary maximum noticeable at $x \approx 1.37$ corresponds to the resonance excitation of the magnetic quadrupolar mode and has nothing to do with the matter discussed.

Let us stress a counterintuitive feature of the presented plots, which is important for applications: At $\theta>90^{\circ}$ the points of the local minima in the directional scattering almost coincide with the point of the local maximum of the overall extinction cross section, e.g., at $\theta=130^{\circ}, x_{\min }^{(\text {dir) }} \approx 1.315, x_{\max }^{\text {(ext) }} \approx 1.331$, respectively.

Discussion. To understand the obtained results, we have to remember that, in the specified range of the size parameter, only the two modes, the electric dipolar and quadrupolar, make the key contribution to the scattered intensity. In this case, in the far zone, the intensity, scattered at a given angle $\theta$ and polarized perpendicular to the scattering plane, is given by the following expression [12],

$$
I_{\mathrm{S}} \approx\left(\frac{\lambda}{2 \pi r}\right)^{2}\left|{ }^{e} B_{1}\right|^{2}|1+f(x) \cos \theta|^{2}
$$

where $r$ is the distance from the scatterer to the observation point in the spherical coordinate frame, whose origin coincides with the one of the scattering sphere, $f(x)$ stands for $-3 i\left({ }^{e} B_{2} /{ }^{e} B_{1}\right)$, and the complex quantities ${ }^{e} B_{1,2}$ (the electric dipolar and quadrupolar coefficients, respectively) are expressed in terms of $x$ and the complex refractive index $\hat{m}=n+i \kappa \equiv \sqrt{\varepsilon}$ through the Riccati-Bessel functions. The corresponding expressions are cumbersome [12], and will not be presented here.

Now note that the partial scattering efficiencies are proportional to the square of the modula of the corresponding scattering coefficients [13]. Then, as it follows from Fig. 1, at the domain that is most essential for the resonances discussed, $1.28 \leqslant x \leqslant 1.36$, the quantity $\left|{ }^{e} B_{1}\right|^{2}$ may be regarded as a constant with quite a reasonable accuracy. In this case, in the specified segment dependence, $I_{\mathrm{S}}(x, \theta)$ is virtually entirely determined by the product $f(x) \cos \theta$. On the other hand, in the discussed range of variations of $x$, the complex quantity $f(x)$ exhibits a typical resonant behavior (see Fig. 4). Then, it may be approximated by a Lorentzian profile,

$$
f(x) \approx A \frac{(\Gamma / 2) e^{i \phi_{0}}}{\left(x-x_{\mathrm{res}}\right)+i(\Gamma / 2)},
$$

where $A, \Gamma$, and $\phi$ are fitting parameters. Their values employed to obtain Fig. 4 are as follows: $A=1.767, \Gamma=$ $0.034, \phi_{0}=2.379, x_{\text {res }}=1.331$.

The substitution of Eq. (2) into Eq. (1) yields the following profile for the scattered intensity,

$$
\left(\frac{2 \pi r}{\left.\lambda\right|^{e} B_{1}\left(x_{\mathrm{res}}\right) \mid}\right)^{2} I_{\mathrm{S}} \approx I_{\epsilon} \equiv \frac{(\epsilon+q)^{2}}{\epsilon^{2}+1}+\frac{\left(1+q \tan \phi_{0}\right)^{2}}{\epsilon^{2}+1}
$$

where $\epsilon \equiv 2\left(x-x_{\text {res }}\right) / \Gamma, q \equiv A \cos \phi_{0} \cos \theta$.

Thus, the obtained profile is a superposition of the conventional Fano line $[3,22]$ [the first term in the right-hand side of Eq. (3)] and the Lorentzian profile (the second term). It is important to note that while the positions of the extrema of the Fano profile (the minimum at $\epsilon=-q$ and maximum at $\epsilon=1 / q$ ) depend on the scattering angle, the position of the maximum of the Lorentzian line is fixed at $\epsilon=0$. In contrast, the amplitude of the Lorentzian profile in Eq. (3) is $\theta$ dependent (at the selected values of the fitting parameters it is a monotonically decreasing function of $\theta$ at the entire range $0 \leqslant \theta \leqslant 130^{\circ}$ ). It means that, by varying $\theta$, we may vary the distance between the minimum of the Fano profile and the maximum of the superimposed Lorentzian one as well 
(a)

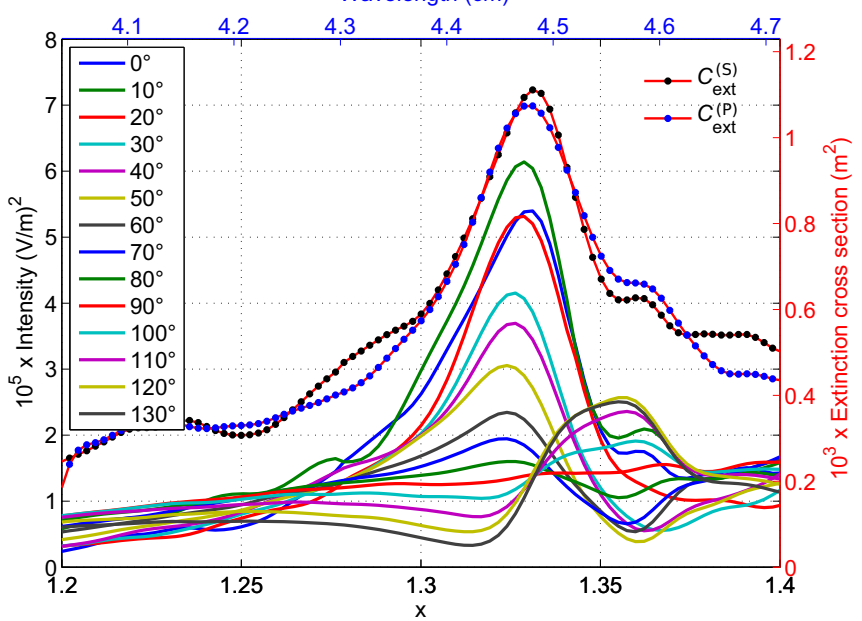

(b)

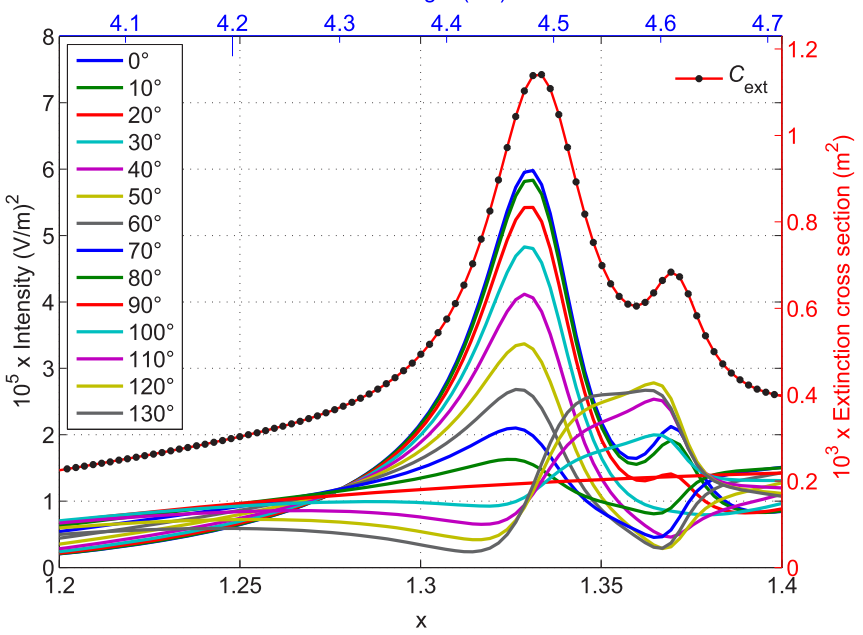

FIG. 3. The intensity of the wave in the $\mathrm{S}$ polarization scattered by the sphere in the azimuthal plane along the angle $\theta\left[I_{\mathrm{S}}(x, \theta)\right]$ and the extinction cross section $C_{\text {ext }}(x)$. (a) Experimentally measured (Meas.). (b) Simulation (Sim.) according to the exact Mie solution. $C_{\text {ext }}(x)$ is measured for both polarizations independently. An increase in $\theta$ results in a monotonic decrease of the local maximum in the vicinity of $x=1.32$ until it vanishes at $\theta=90^{\circ}$. A further increase in $\theta$ transforms it into a local minimum, whose value continues to decrease with an increase in $\theta$. The case with the local minimum in the vicinity of $x=1.36$ is opposite.

as the relative role of the contribution of the Lorentzian profile, controlling the sharpness of the corresponding part of $I_{\epsilon}$ and the amplitude of its modulations.

To illustrate the description of the actual line shapes by the approximation (3), the corresponding profiles for $\theta$ varying from 0 to $130^{\circ}$ in steps of $10^{\circ}$ (the same range and sampling as those in Fig. 3) are presented in Fig. 5. The values of the fitting parameters are the same as those employed to plot Fig. 4. For an easier comparison of Fig. 3 with Fig. $5, I_{\epsilon}$ in the latter is plotted as a function of $x$. The difference between the right wings of the lines in Figs. 5 and 3 is explained by the departure

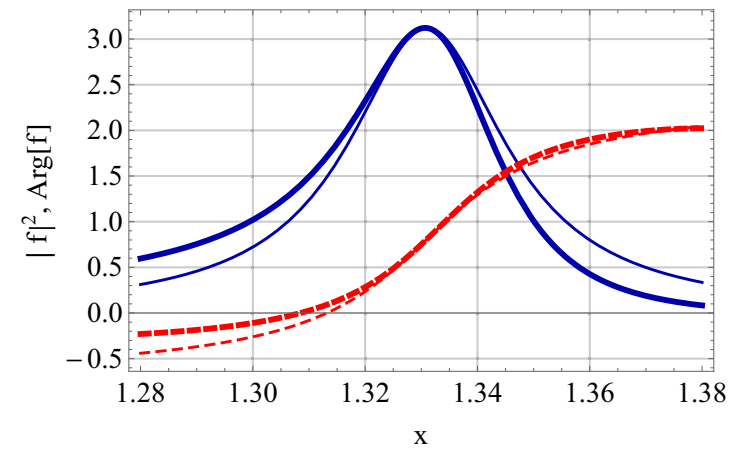

FIG. 4. Modulus (blue solid lines) and phase (red dashed lines) of complex function $f(x) \equiv-3 i\left({ }^{e} B_{2} /{ }^{e} B_{1}\right)$ at $\varepsilon=17.2+0.2 i$ calculated according to the exact Mie solution (thick lines). Its fit by approximation (2) is shown with the corresponding thin lines.

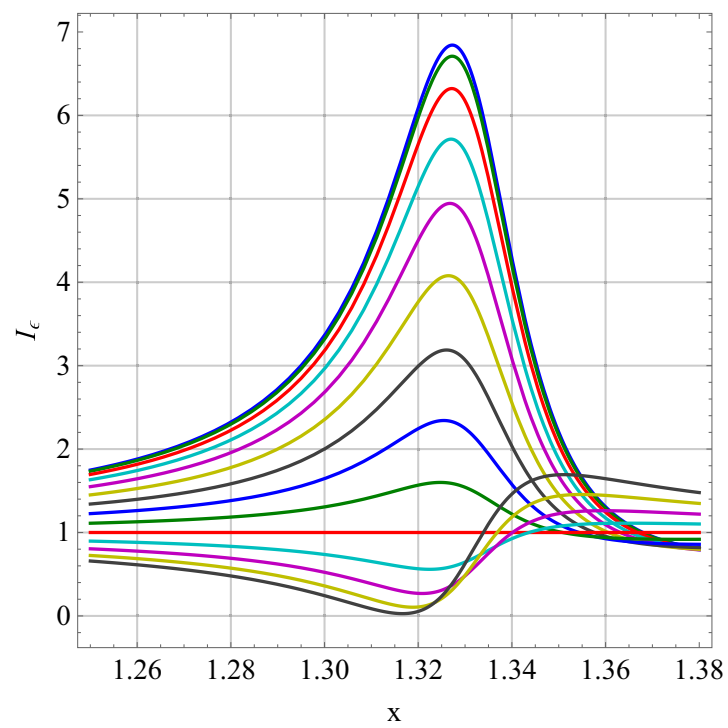

FIG. 5. $I_{\epsilon}$ according to Eq. (3) for $\theta$ varying from 0 to $130^{\circ}$ in steps of $10^{\circ}$ as a function of $x$ (cf. Fig. 3).

in this range of approximation (2) for $f(x)$ from the actual profile, obtained from the Mie solution, and the contribution of the magnetic quadrupolar mode, which is not accounted for in Eq. (1) [23]. Apart from this point, the agreement between the two sets of figures is quite impressive.

As for the shape of the net scattering cross section, the latter is a sum of positive contributions of every partial one. In turn, the partial cross sections are proportional to the squares of the modula of the corresponding scattering coefficients [13]. In the discussed range of variations of the size parameter, just two coefficients, ${ }^{e} B_{1}$ and ${ }^{e} B_{2}$, make an overwhelming contribution to the net cross section. Moreover, we have shown that, in this range, $\left|{ }^{e} B_{1}\right|^{2}$ may be regarded as a constant while $\left|{ }^{e} B_{2}\right|^{2}$ has a Lorentzian shape (see Figs. 1 and 4). These arguments guarantee the Lorentzian shape of the net scattering cross section in the discussed domain of variations of $x$. The same is true for the extinction cross section since the difference between the two is negligibly small owing to the weak dissipation. 
Conclusions. In this Rapid Communication, due to the microwave analogy principle, we have mimicked the scattering of linearly plane polarized light in the visible and near IR range by a homogeneous nanosphere made of a common semiconductor with the scattering of a macroscopic ceramic sphere. This approach makes it possible to obtain detailed experimental results on the dependences of the scattering intensity on the scattering angle and the size parameter as well as the line shape for the net extinction cross section. We have found and employed such a range of the size parameter where just the two modes, electric dipolar and quadrupolar, make an overwhelming contribution to the net scattering cross section and shown that the interference of these two partial waves gives rise to directional Fano resonances. At the same time, the line shapes of the net extinction and scattering cross sections in this range remain Lorentzian. We have obtained a simple analytical expression for the line shape of the intensity scattering along any given direction and shown that it consists of two superimposed profiles. One of them is a conventional Fano profile, while the other is Lorentzian. For the Fano profile, the values of the extrema and their positions both depend on the scattering angle, while for the Lorentzian profile only the value of its maximum depends on this quantity, while its position is fixed. It provides additional opportunities to tailor and engineer the line shape by varying the scattering angle. The latter may be important to modify a desired line shape for practical applications of the discussed effects in sensors and analogous devices.

Acknowledgments. This research has been supported by MICINN (Spanish Ministry of Science and Innovation, Project No. FIS2013-45854-P). We also acknowledge the opportunity provided by the Centre Commun de Ressources en Microonde to use its fully equipped anechoic chamber.
[1] M. I. Mishchenko, L. D. Travis, and A. A. Lacis, Scattering, Absorption, and Emission of Light by Small Particles (Cambridge University Press, Cambridge, UK, 2002).

[2] B. Luk'yanchuk, N. I. Zheludev, S. A. Maier, N. J. Halas, P. Nordlander, H. Giessen, and C. T. Chong, Nat. Mater. 9, 707 (2010).

[3] A. E. Miroshnichenko, S. Flach, and Yu. Kivshar, Rev. Mod. Phys. 82, 2257 (2010).

[4] M. I. Tribelsky, S. Flach, A. E. Miroshnichenko, A. V. Gorbach, and Y. S. Kivshar, Phys. Rev. Lett. 100, 043903 (2008).

[5] M. V. Rybin, P. V. Kapitanova, D. S. Filonov, A. P. Slobozhanyuk, P. A. Belov, Y. S. Kivshar, and M. F. Limonov, Phys. Rev. B 88, 205106 (2013).

[6] J. Yan, P. Liu, Z. Lin, H. Wang, H. Chen, C. Wang, and G. Yang, ACS Nano 9, 2968 (2015).

[7] M. Kerker, D. S. Wang, and L. Giles, J. Opt. Soc. Am. 73, 765 (1983).

[8] R. Vaillon and J.-M. Geffrin, J. Quant. Spectrosc. Radiat. Transfer 146, 100 (2014).

[9] J.-M. Geffrin, B. García-Cámara, R. Gómez-Medina, P. Albella, L. S. Froufe-Pérez, C. Eyraud, A. Litman, R. Vaillon, F. González, M. Nieto-Vesperinas, J. J. Sáenz. and F. Moreno, Nat. Commun. 3, 1171 (2012).

[10] M. Rybin, D. Filonov, K. Samusev, P. A. Belov, Y. S. Kivshar, and M. F. Limonov, Nat. Commun. 6, 10102, (2015).

[11] Eccostock-HIK from Emerson and Cuming, http://www.eccosorb.com/.

[12] M. Born and E. Wolf, Principles of Optics (Cambridge University Press, Cambridge, UK, 1999).
[13] C. F. Bohren and D. R. Huffman, Absorption and Scattering of Light by Small Particles (Wiley, New York, 1998).

[14] C. Eyraud, J.-M. Geffrin, A. Litman, and H. Tortel, IEEE Antennas Wireless Propag. Lett. 14, 309 (2015).

[15] A. R. Forouhi and I. Bloomer, Phys. Rev. B 38, 1865 (1988).

[16] M. I. Tribelsky, J.-M. Geffrin, A. Litman, C. Eyraud, and F. Moreno, Sci. Rep. 5, 12288 (2015).

[17] J.-M. Geffrin, C. Eyraud, A. Litman, and P. Sabouroux, Radio Sci. 44, RS2007 (2009).

[18] J.-M. Geffrin, C. Eyraud, and A. Litman, IEEE Microwave Wireless Compon. Lett. 25, 472 (2015).

[19] O. M. Bucci and G. Franceschetti, IEEE Trans. Antennas Propag. 35, 1445 (1987).

[20] C. Eyraud, J.-M. Geffrin, A. Litman, P. Sabouroux, and H. Giovannini, Appl. Phys. Lett. 89, 244104 (2006).

[21] C. Larsson, C. Sohl, M. Gustafsson, and G. Kristensson, in Proceedings of the 3rd European Conference on Antennas and Propagation (EuCAP) 2009 (IEEE, Piscataway, NJ, 2009), p. 3633.

[22] U. Fano, Phys. Rev. 124, 1866 (1961).

[23] Strictly speaking, we have to compare the profile $I_{\epsilon}(x)$ with $x^{2} I_{\mathrm{S}}(x)$ [see Eq. (3)]. However, the substantial parts of the profiles correspond to variations of $x$ from 1.28 to 1.36 (see Figs. 3-5). In this domain, $x^{2} I_{\mathrm{S}}(x) \approx x_{\text {res }}^{2} I_{\mathrm{S}}(x)$ with an accuracy of a few percent, and the line shape of $x^{2} I_{\mathrm{S}}(x)$ almost coincides with that for $I_{\mathrm{S}}(x)$. Note also that the weak quadrupole resonance at the vicinity of $x=1.37$ exhibits its own directional Fano profiles. These profiles are superimposed with the discussed dipolar ones, enhancing the asymmetry of the right wing of the overall scattering line. 